EPJ Web of Conferences 60, 10003 (2013)

DOI: $10.1051 /$ epjconf/20136010003

(c) Owned by the authors, published by EDP Sciences, 2013

\title{
ALICE Upgrades
}

\author{
Thomas Peitzmann ${ }^{1, a}$ (for the ALICE Collaboration) \\ ${ }^{1}$ Nikhef Amsterdam and Utrecht University, The Netherlands.
}

\begin{abstract}
While groundbreaking measurements on the properties of strongly interacting matter in $\mathrm{p}+\mathrm{p}, \mathrm{p}+\mathrm{A}$ and $\mathrm{A}+\mathrm{A}$ collisions at the LHC are being performed, it is clear that many important questions in heavy-ion physics will remain unanswered in this first phase of beam times up to 2017. The ALICE collaboration is setting up a program of detector upgrades to be installed in the LHC shutdown planned for 2017/18, to address the new scientific challenges. We will discuss examples of the scientific frontiers and upgrade projects under study for the ALICE experiment.
\end{abstract}

\section{Introduction: the physics frontiers for ALICE}

In the first years of operation ALICE has demonstrated its excellent capabilities to measure high-energy nuclear collisions at the LHC, delivering exciting results on elliptic flow, which appears to be even larger than at RHIC [1], strong quenching of high momentum hadrons including first measurements using identified open charm mesons [2] and intriguing results on the centrality dependence of charmonium production [3], to just name a few examples. Further data taking up to 2017 will improve the understanding of heavy-ion collisions significantly and will very likely yield comprehensive results on many experimental probes, as e.g. the details of anisotropic flow of light hadrons, inclusive momentum spectra of heavy flavour mesons including their nuclear modification, or the global features of jets in nuclear collisions, all-in-all the baseline program of the ALICE experiment.

Still, crucial measurements will not be possible. For illustration let us consider the study of collective motion of charm. While the measurement of spectra of open charm mesons is challenging, it has already been shown to be feasible, and a further increase in statistics to $0.1 \mathrm{nb}^{-1}$ (or $1 \mathrm{nb}^{-1}$ for rare triggered probes) should allow precision measurements of the most abundant D mesons. However, anisotropic flow studies generally require an order of magnitude higher statistics, thus calling for $\approx 10 \mathrm{nb}^{-1}$ in $\mathrm{Pb}$ $\mathrm{Pb}$ collisions. In addition, it is known from light hadrons, that significant differences essential for theoretical understanding of collective flow are observed between mesons and baryons. This requires a measurement of charmed baryons, as e.g. the $\Lambda_{c}$. Furthermore, it is crucial for studies of equilibration properties to cover the lowest transverse momenta possible. Both, the measurement of the $\Lambda_{c}$, and a significant measurement at very low $p_{T}$ will

\footnotetext{
a e-mail: t.peitzmann@uu.nl
}

profit enormously from an improved background rejection and secondary vertex resolution of the tracking detectors. Similar arguments hold for other important physics signals of the quark-gluon plasma, that will not be accessible with the current apparatus and the available integrated luminosity up to the long shutdown in 2017/18. Overall, an upgraded ALICE setup at high luminosity should allow

1. precision studies of primary charm (open and hidden) including flow and correlations,

2. measurements of low mass lepton pairs and thermal photons,

3. studies of jet modification via gamma-jet and jet-jet correlations including particle ID, and

4. the search for heavy nuclear states.

ALICE is preparing an upgrade program that builds on the existing strengths of the experimental setup, such as excellent tracking performance, in particular at low momenta, efficient secondary vertex reconstruction, very low material budget, and excellent particle identification. The upgraded ALICE detector will significantly improve the performance in most of these areas, except for particle identification, which will be preserved. Furthermore, the upgraded experimental setup will have enhanced rate capabilities to allow an inspection of $\mathrm{Pb}-\mathrm{Pb}$ collisions at an interaction rate of $50 \mathrm{kHz}$. This is necessary to be able to exploit the physics potential, because many of the signals have an irreducibly low signal-to-background ratio requiring a high statistics measurement, but at the same time making them untriggerable. Fortunately, the signalto-background for these observables will be much better in pp, such that only a moderately higher luminosity $(\approx 200 \mathrm{kHz})$ will be needed for reference measurements. At appropriate luminosity the upgraded apparatus will allow us to collect sufficient reference statistics in short reference runs. Crucial parts of the upgrade are the develop- 
Table 1. Comparison of the physics performance for selected observables between the approved scenario $\left(1 \mathrm{nb}^{-1}\right.$ of delivered integrated luminosity, out of which $0.1 \mathrm{nb}^{-1}$ is used for minimum-bias data collection) and the proposed upgrade (10 $\mathrm{nb}^{-1} \mathrm{of}^{-}$ integrated luminosity). Given are minimum accessible $p_{T}$ and relative statistical uncertainty.

\begin{tabular}{|c|c|c|c|c|}
\hline \multirow[b]{2}{*}{ Observable } & \multicolumn{2}{|c|}{ Approved } & \multicolumn{2}{|r|}{ Upgrade } \\
\hline & $\begin{array}{c}p_{\mathrm{T}}^{\mathrm{Amin}} \\
(\mathrm{GeV} / c)\end{array}$ & $\begin{array}{c}\text { statistical } \\
\text { uncertainty }\end{array}$ & $\begin{array}{c}p_{\mathrm{T}}^{\mathrm{Umin}} \\
(\mathrm{GeV} / c)\end{array}$ & $\begin{array}{c}\text { statistical } \\
\text { uncertainty }\end{array}$ \\
\hline D meson from B decays $R_{\mathrm{AA}}$ & 3 & $30 \%$ at $p_{\mathrm{T}}^{\text {Amin }}$ & 2 & $1 \%$ at $p_{\mathrm{T}}^{\text {Amin }}$ \\
\hline D meson elliptic flow $\left(v_{2}=0.2\right)$ & 1 & $50 \%$ at $p_{\mathrm{T}}^{\text {Amin }}$ & 0 & $2.5 \%$ at $p_{\mathrm{T}}^{\text {Amin }}$ \\
\hline Charm baryon-to-meson ratio & \multicolumn{2}{|c|}{ not accessible } & 2 & $15 \%$ at $p_{\mathrm{T}}^{\mathrm{Umin}}$ \\
\hline $\mathrm{J} / \psi R_{\mathrm{AA}}$ (forward rapidity) & 0 & $1 \%$ at $1 \mathrm{GeV} / c$ & 0 & $0.3 \%$ at $1 \mathrm{GeV} / c$ \\
\hline $\mathrm{J} / \psi R_{\mathrm{AA}}$ (mid-rapidity) & 0 & $5 \%$ at $1 \mathrm{GeV} / c$ & 0 & $0.5 \%$ at $1 \mathrm{GeV} / c$ \\
\hline$\psi(2 \mathrm{~S})$ yield & 0 & $30 \%$ & 0 & $10 \%$ \\
\hline Low-mass $e^{+} e^{-}$spectral function & \multicolumn{2}{|c|}{ not accessible } & 0.3 & $20 \%$ \\
\hline
\end{tabular}

Table 2. Possible running scenario for the operation of the upgraded ALICE detector.

\begin{tabular}{llll}
\hline year & beams & int. luminosity & remarks \\
\hline 2019 & $\mathrm{~Pb}-\mathrm{Pb}$ & $2.85 \mathrm{nb}^{-1}$ & \\
2020 & $\mathrm{~Pb}-\mathrm{Pb}$ & $2.85 \mathrm{nb}^{-1}$ & low magnetic field \\
2021 & $\mathrm{pp}$ & t.b.c. & reference run \\
2022 & - & - & long shutdown 3 \\
2023 & - & - & long shutdown 3 \\
2024 & $\mathrm{~Pb}-\mathrm{Pb}$ & $2.85 \mathrm{nb}^{-1}$ & \\
2025 & $\mathrm{~Pb}-\mathrm{Pb}$ & $1.42 \mathrm{nb}^{-1}$ & $50 \%$ of beamtime \\
& $\mathrm{p}-\mathrm{Pb}$ & $50 \mathrm{nb}^{-1}$ & $50 \%$ of beamtime \\
2026 & $\mathrm{~Pb}-\mathrm{Pb}$ & $2.85 \mathrm{nb}^{-1}$ & \\
\hline
\end{tabular}

ment and implementation of a significantly improved inner tracking system (ITS), the replacement of the TPC readout chambers with GEMs, a general upgrade of the trigger detectors (e.g. V0, T0) and of all readout electronics, and a new system integrating DAQ, High-Level Trigger and offline computing. Examples for the performance improvement for important observables are given in Table 1. More information on the improvements and further details on the ALICE upgrade can be found in [4].

Another new detector, the Muon Forward Tracker, is being proposed to possibly further enhance the measurement capabilities for the signals mentioned above via the improved forward measurement of quarkonia and lowmass dimuons, as well as heavy-flavour muons. Installation of these detector upgrades is foreseen for the LHC long shutdown 2, implying a measurement program for $\mathrm{pp}$, $\mathrm{p}-\mathrm{Pb}$, and $\mathrm{Pb}-\mathrm{Pb}$ extending up to $\approx 2026$ - a possible scenario for the measurement program is summarised in Table 2 .

Additionally, with an appropriate detector at large rapidity an opportunity arises for measurements, where the influence of small- $x$ partons is more and more important. This is the region, where effects of gluon saturation [5] should be most prominent. Signals consistent with gluon saturation have been observed at RHIC [6], but these remain inconclusive, because the interpretation is hampered by the very limited kinematical reach. The larger beam energy of the LHC will allow us to enter a new physics regime with access to much smaller values of $x$ and a larger phase space for saturation due to the expected larger saturation scale.

\section{ALICE rate capabilities and the upgraded ITS}

The existing Time Projection Chamber (TPC) [7] is a unique element of the current ALICE apparatus with its continuous tracking and particle identification. However, with its long drift time it is limiting the rate capabilities. Using the multi-wire readout chambers, a gated operation (at a maximum of $3.5 \mathrm{kHz}$ ) is necessary to limit ion feedback to the drift volume, which would otherwise lead to intolerable space-charge distortions. To be able to inspect a significantly higher interaction rate, new readout chambers based on triple-GEMs will be developed. These would be continuously read out (no gating) and should have small enough ion feedback to allow an operation at $50 \mathrm{kHz}$ interaction rate. $R \& D$ on the GEM readout chambers is ongoing with an emphasis on achieving the projected low ion feedback and in particular on demonstrating the necessary stability in a high multiplicity/high rate environment. In a pipelined readout data would be shipped from the TPC front-end at $10 \mathrm{MHz}$ for later inspection in a high level trigger system (HLT). The readout electronics of all existing detector systems will be modified to accommodate the rate capabilities. Some systems will likely share the pipelined electronics with the TPC, other detectors, e.g. the electromagnetic calorimeter (EMCal), might preferably be readout on L1 triggers provided by a hardware trigger unit.

The high rate upgrade scenario results in $\approx 1 \mathrm{~TB} / \mathrm{s}$ of input to the online systems, where most of the data originates from the TPC readout. This necessarily will be reduced in particular for the TPC information via cluster 


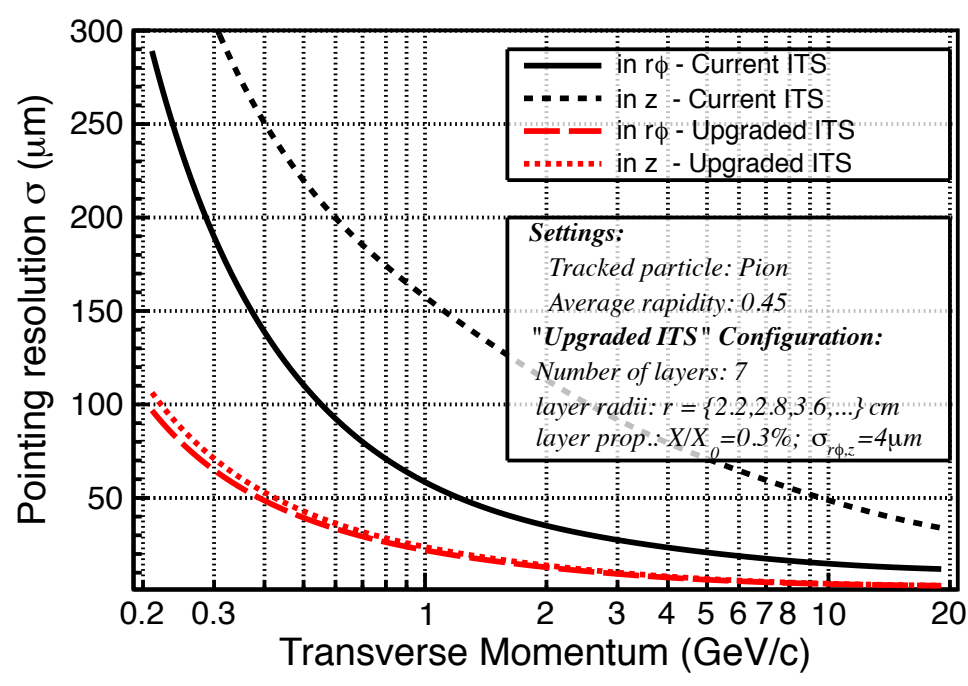

Figure 1. Track impact parameter resolution in $r \phi$ and $z$ for the current (black) and upgraded (red) ITS.

finding and cluster-track association, as well as standard Huffman encoding. An average output rate to tape storage of the order of $10 \mathrm{~GB} / \mathrm{s}$ after this data compression should be achievable.

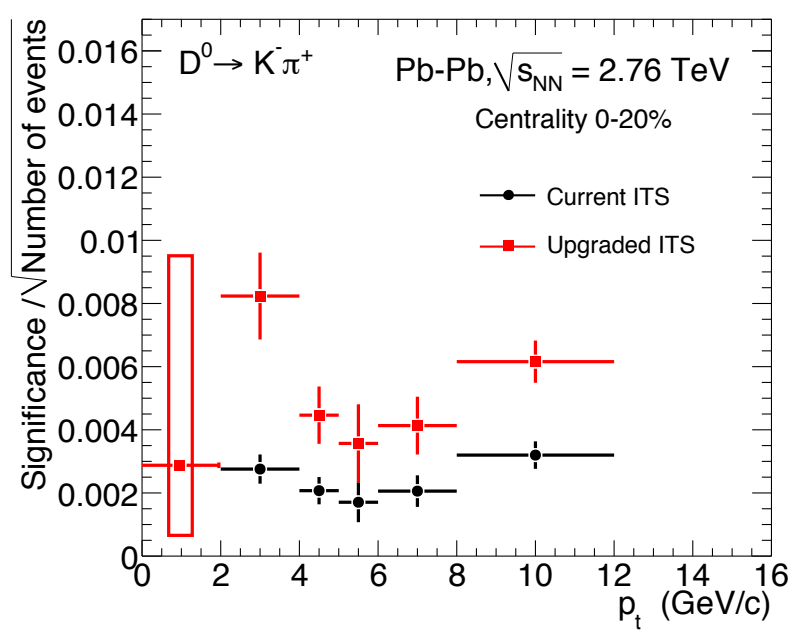

Figure 2. Significance for the measurement of the $D^{0}$ as a function of $p_{T}$ normalised to the square root of the number of events. The performance of the upgraded ITS is compared to that of the current detector.

A completely new Inner Tracking System (ITS) will be developed. The most important features of the new design in addition to enhanced rate capabilities will be:

- A decrease of the radial distance of the first layer to the beam to $22 \mathrm{~mm}$ (from $39 \mathrm{~mm}$ ),

- a reduction of the layer thickness possibly down to 50 $\mu \mathrm{m}$ (from $350 \mu \mathrm{m}$ ), and

- a higher granularity with pixel sizes as small as $20 \times 20$ $\mu \mathrm{m}^{2}$.
This should be achievable by implementing seven layers of Monolithic Active Pixel Sensors and further optimisation of cabling, mechanical and cooling structures.

The new ITS will improve the secondary vertex resolution by a factor of $\approx 3$ (see Fig. 1) and allow measurements down to much lower values of $p_{T}$. Furthermore the high efficiency and low contamination of the new ITS should allow for enhanced level 2 trigger capabilities. It should give access to the measurement of charmed and beauty baryons and to the discrimination of prompt and secondary heavy quark production.

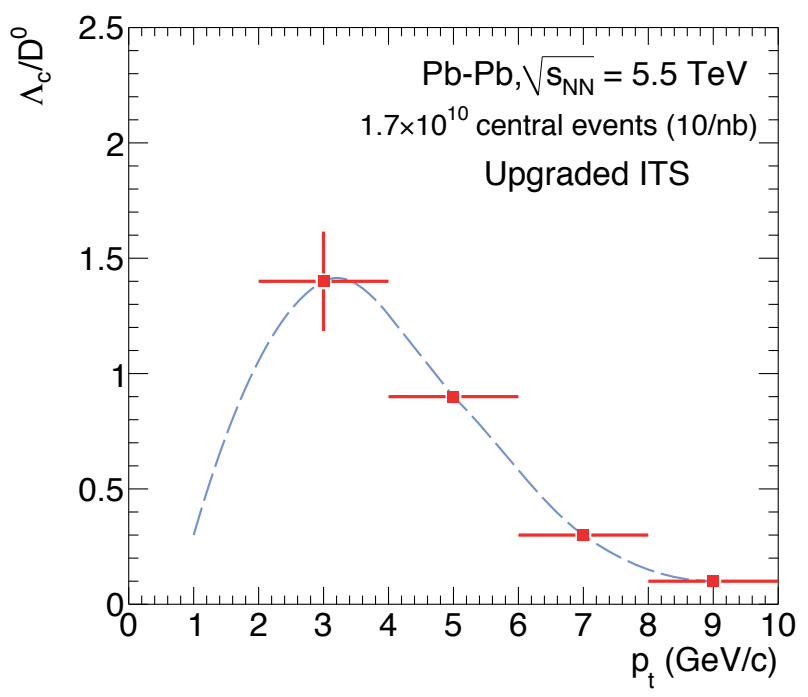

Figure 3. Possible measurement with the upgrade of the $\Lambda_{c} / D^{0}$ ratio as a function of $p_{T}$ for central $\mathrm{Pb}-\mathrm{Pb}$ collisions. The points are drawn on a line that captures the behaviour of the measured $\Lambda / K_{S}^{0}$ ratio.

Fig. 2 shows a comparison of the expected significance for a $D^{0}$ measurement with the upgraded ITS to that expected with the current detector - in particular at low $p_{T}$ 

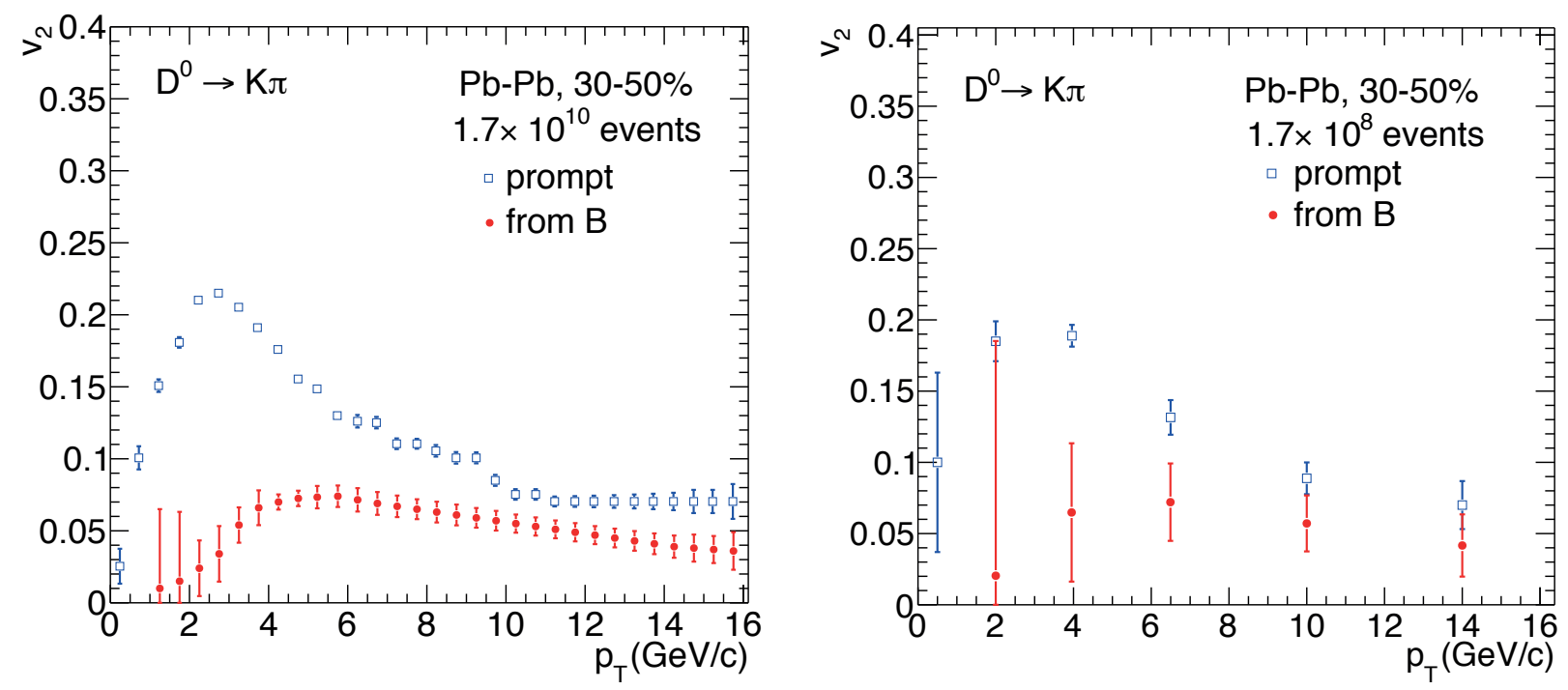

Figure 4. Estimated statistical uncertainties on $v_{2}$ of prompt and secondary $\mathrm{D}^{0}$ mesons for $1.7 \cdot 10^{10}$ events (left) in the $30-50 \%$ centrality class, which correspond to $10 \mathrm{nb}^{-1}$, and for $1.7 \cdot 10^{8}$ events (right), which correspond to about $0.1 \mathrm{nb}^{-1}$.

the performance improves significantly. Even more crucial is the upgrade for a measurement of the $\Lambda_{c}$ baryon in heavy-ion collisions, which cannot be measured with the current apparatus. In Fig. 3 one can see the expected statistical uncertainty for a measurement of the $\Lambda_{c} / D^{0}$ ratio in central $\mathrm{Pb}-\mathrm{Pb}$ collisions with the upgrade.

As another important example of the estimated performance Fig. 4 shows simulations of an elliptic flow measurement of prompt and secondary D mesons for $10 \mathrm{nb}^{-1}$ (left) and for $0.1 \mathrm{nb}^{-1}$ (right), the former of which would only be accessible with the upgrade.

The improved background rejection and the better identification of heavy-flavour electrons would also enable us to perform unprecedented measurements of low-mass dileptons - these would profit further from a dedicated run at lower magnetic field to improve the detector acceptance at low mass and low $p_{T}$. More details on the design and the physics program and performance of the new ITS is described in [8].

\section{Additional Detector Upgrades}

Beyond the above upgrade programme, which has been endorsed by the LHC experiments committee (LHCC), further upgrades are planned. In particular, ALICE is proposing to improve the physics performance of the muon measurements at forward rapidities $(-4.0<\eta<$ -2.5). The Muon Forward Tracker (MFT) will be located upstream of the hadron absorber of the Muon Spectrometer, close to the new ITS, and will consist of five tracking planes built on the same monolithic active pixel technology as the new ITS. By adding tracking in front of the muon absorber, it would allow us to reconstruct possible secondary vertices of muon tracks. This would remove the largest limitation of the current forward muon measurements, enabling the identification of $J / \psi$ from $B$ decays and would in general improve the resolution and signal-tobackground ratio resulting in better general performance for measurements of quarkonia and low mass dileptons. E.g., the addition of the MFT would improve the signalto-background ratio and systematic errors for the measurement of the $\psi^{\prime}$ in central $\mathrm{Pb}-\mathrm{Pb}$ collisions by a factor of 3 10 depending on the transverse momentum. The expected improvement for the measurement of the corresponding nuclear modification factor with the MFT compared to the performance of the existing muon arm alone is illustrated in Fig. 5.

Studies are ongoing for a Forward Calorimeter (Fo$\mathrm{Cal}$ ) for photon and possibly jet measurements in the forward region $(\eta \approx 3-5)$ opposite the Muon Spectrometer. The project is still being discussed within the collaboration, and thus installation will not be possible on the same time scale as for the upgrades discussed above. While the demands on energy resolution are moderate for forward measurements, the small opening angle of neutral pion decays and the overall large particle density in $\mathrm{Pb}-\mathrm{Pb}$ collisions will require good position resolution and two-particle separation power. The idea is to implement an electromagnetic calorimeter with small Molière radius and extreme granularity, most likely a SiW sandwich design with cell sizes of $\approx 1 \mathrm{~mm}^{2}$, which will allow a precise direct photon measurement. For the high-granularity sensors, the same MAPS technology as for ITS and MFT is being considered. This detector would likely be complemented by a conventional hadronic calorimeter, that would improve jet reconstruction and isolation cuts for the photon measurements. The detector would allow a detection of neutral pions and direct photons in an $\eta$ and $p_{T}$ range not accessible to existing experiments, and would give unprecedented sensitivity to small- $x$ gluon structure and saturation effects. 

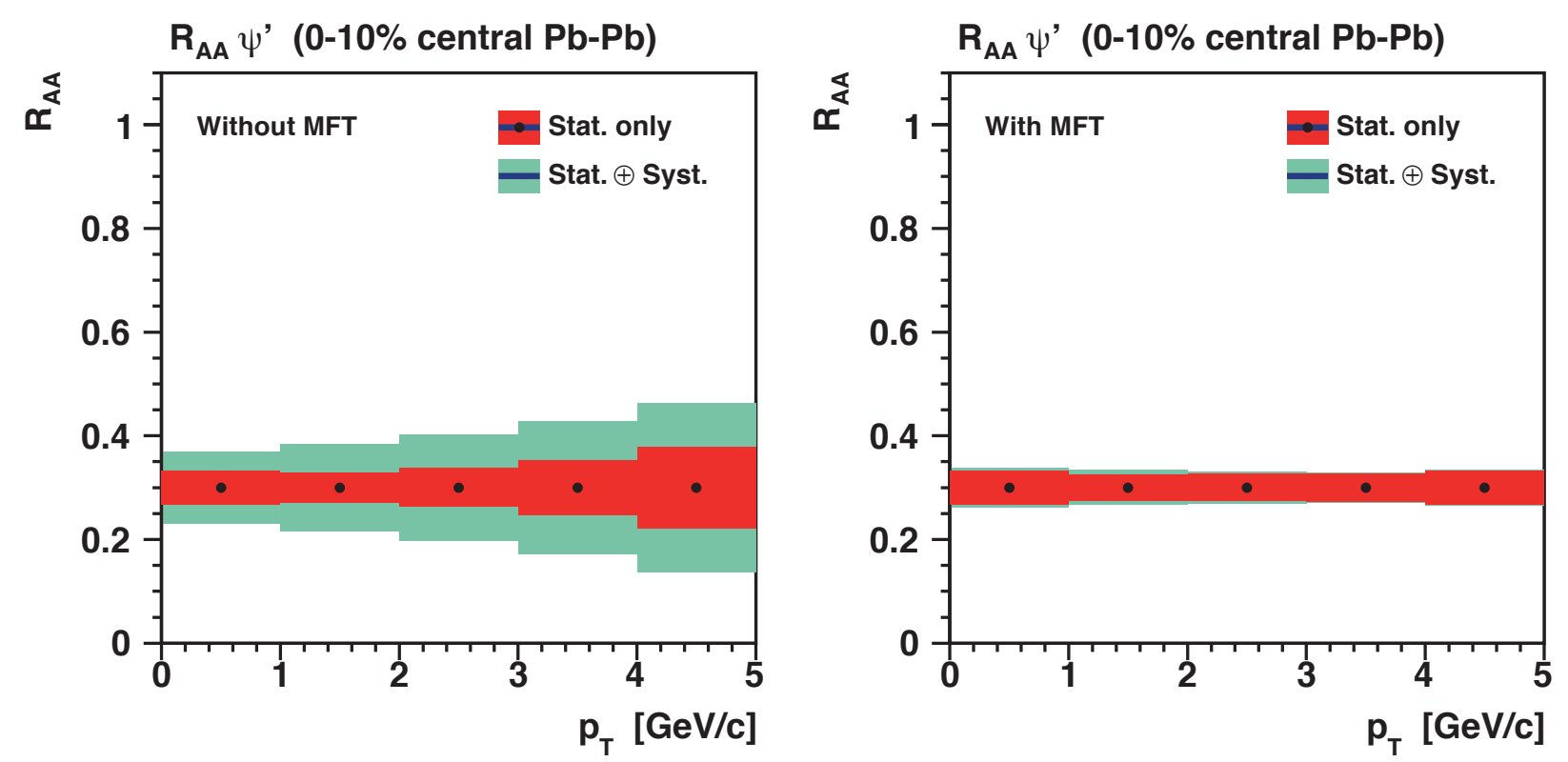

Figure 5. Expected statistical and systematical uncertainties of the nuclear modification factor $R_{A A}$ of the $\psi^{\prime}$ in central $\mathrm{Pb}-\mathrm{Pb}$ collisions without MFT (left panel) and with MFT (right panel).

\section{References}

[1] K. Aamodt et al. (ALICE), Phys. Rev. Lett. 105, 252302 (2010).

[2] B. Abelev et al. (ALICE), JHEP 09, 112 (2012).

[3] B. Abelev et al. (ALICE), Phys. Rev. Lett. 109, 072301 (2012).

[4] ALICE Collaboration, Upgrade of the ALICE Experiment - Letter Of Intent, CERN-LHCC-2012-012 / LHCC-I-022.

[5] L.V. Gribov, E.M. Levin, and M.G. Ryskin, Phys. Rept. 100, 1 (1983); A.H. Mueller and J.-W. Qiu, Nucl.
Phys. B 268, 427 (1986); L.D. McLerran and R. Venugopalan, Phys. Rev. D 50, 2225 (1994); Phys. Rev. D 49, 3352 (1994).

[6] I. Arsene et al. (BRAHMS), Phys. Rev. Lett. 93, 242303 (2004); J. Adams et al. (STAR), Phys. Rev. Lett. 97, 152302 (2006).

[7] K. Aamodt et al. (ALICE), JINST 3, S08002 (2008).

[8] ALICE Collaboration, Upgrade of the Inner Tracking System - Conceptual Design Report, CERN-LHCC2012-013 / LHCC-P-005.

[9] C.A. Salgado, J. Phys. G 38, 124036 (2011). 